CALT-68-2010

\title{
Optical scattering angles and Bose-stimulated motion in cold atomic gas
}

\author{
H. David Politzer \\ California Institute of Technology, Pasadena, California 91125 \\ politzer@theory.caltech.edu
}

(October 6, 1995)

\begin{abstract}
Bose statistics imply a substantial enhancement at small angles for light scattering off a cold, Bose gas. The enhancement increases dramatically at the Bose-Einstein temperature. This phenomenon could be utilized to eliminate almost entirely the heating of the gas by a weak probe light beam.
\end{abstract}

PACS numbers 03.75.Fi, 05.30.Jp, 32.80.Pj, 42.50.Gy

Typeset using REVTEX 
A salient feature of Bose statistics is that the transition probability to a particular final state is proportional to $n_{f}+1$, where $n_{f}$ is the number of bosons hitherto in that final state. For photons, this is the origin of stimulated emission due to the presence of an external electromagnetic field. The analog for the motion of bosonic atoms is the enhancement of scattering into already occupied states. This is the essential ingredient in the dynamics of the formation of a Bose-Einstein condensate as the temperature drops below the critical value, $T_{\mathrm{c}}$. A boson transition probability is also linear in the number of identical particles in the initial state. The present note points out how these factors effect simple photon-atom scattering in the Born approximation (e.g. for weak light). At low temperatures, there is substantial enhancement for small photon scattering angles (corresponding to small momentum transfers) from stimulated scattering of atoms into already occupied low momentum states. Below $T_{\mathrm{c}}$, this enhancement dramatically shifts to even smaller angles due to the contribution of transitions from occupied atomic momentum $\mathbf{p} \neq \mathbf{0}$ states to the Bose condensate $\mathbf{p}=\mathbf{0}$ state and vice versa. Aside from seeing Bose statistics in action, this may have a practical implication for the optical investigation of cold atoms. The incident light intensity - and consequent total heating of the gas - can be reduced by several orders of magnitude for a given, desired signal strength by putting the detector at very small angle. This may allow non-destructive measurements on super-cold gases.

I treat here the simplest case of a uniform medium and study the photon scattering rate per unit time and volume into a given solid angle. A further simplifying approximation is that the atoms are treated as an ideal, i.e. non-interacting, Bose gas, so that the multiparticle states are described by occupancies of single particle states. Whether appropriate in detail to a particular atom trap depends on trap shape and size, atomic density, and beam wavelength and size. However, the same qualitative features will emerge [1].

The underlying process is just Rayleigh scattering (or perhaps on resonance to enhance the rate). I focus on the angular dependence of the rate for fixed incident wavelength. With very sub-Doppler temperatures $T$ (and with a very narrow light frequency band if using a resonance - narrow compared to the atomic line width), there is no relevant energy 
dependence in the scattering amplitude even though we sum over possible initial and final atomic kinetic energies. This is because the range of energies involved in the thermal sum over atoms is negligible compared to the energy scale of variation of the scattering amplitude. The only angular dependence in the underlying "unstimulated" rate comes from the photon polarizations: $\cos ^{2} \theta$ for polarizations in the scattering plane, 1 for perpendicular, $\frac{1}{2}(1+$ $\cos ^{2} \theta$ ) for unpolarized - all of which I treat as 1 for small angles. (The polarization difference may have some potential interest because it vanishes as $\theta \rightarrow 0$ and, hence, weights angles differently.)

For a given, pure, many-particle quantum state with $n_{i}$ atoms in an initial one-particle state and $n_{f}$ atoms initially in the one-particle state into which one of the $n_{i}$ is scattered, the scattering rate is proportional to $n_{i}\left(n_{f}+1\right)$. The thermally averaged rate is, therefore, proportional to $\left\langle n_{i}\left(n_{f}+1\right)\right\rangle$, where the brackets denote the thermal expectation. For $i \neq f$, which is guaranteed by having $\theta \neq 0$, this is equal to $\left\langle n_{i}\right\rangle\left(\left\langle n_{f}\right\rangle+1\right)$ if we ignore interactions between the atoms themselves. Hence, the needed thermal information is simply the thermal average occupancies of the single particle states $i$ and $f$. It is simplest to evaluate these in terms of a chemical potential, which is then adjusted to produce the desired mean total number of particles. Note, however, that for bosons at very low temperatures, if any state has a large occupancy, the expected fluctuations in the occupancy are comparably large. The actual, physical situation of interest will determine which description (fixed particle number or fixed chemical potential) is more convenient.

It is useful to divide the scattering rate into three terms. (a) The total "unstimulated" rate, i.e., the 1 term in $n_{f}+1$, is normalized to $N_{\text {total }} \cdot 1$, where $N_{\text {total }}$ is the total target atom density and where the Rayleigh rate is normalized to 1. (b) The scattering from $p \neq 0$ occupied states to $p \neq 0$ occupied states is represented by a double integral over two Bose thermal occupation factors $(\cdot 1)$. And $(c)$ below $T_{c}$, there is scattering from $p \neq 0$ occupied states to the $p=0$ state, with occupation $N_{o}(\tau)$, and vice versa; these two pieces give identical contributions. Because the integrals over $p$ 's converge as $p \rightarrow 0$, they do not account for a macroscopic occupancy of the $p=0$ state, which must be treated separately. 
Of course, there is no $N_{o} \cdot N_{o}$ term at non-zero angle. Note that the "unstimulated" rate is linear in the density, while the stimulated terms are quadratic.

Choose units such that $\hbar=c=1$. The convenient third choice of units is $m c^{2} k_{B} T_{c}=1$, where $m$ is the atom mass. Let $\Delta$ be the dimensionless measure of the momentum transfer to the photon, i.e. expressed in the natural units of the problem. Then

$$
\Delta=|\Delta|=\frac{\sin \theta}{\cos \frac{\theta}{2}} \frac{h \nu}{\sqrt{m c^{2} k_{B} T_{c}}} \simeq \theta \cdot k
$$

where $\theta$ is the photon scattering angle, $k$ is the magnitude of its momentum in these units, and $\nu$ is its frequency. For laser light scattering off alkali atoms condensing at temperatures of order $10^{-7} \mathrm{~K}, \theta$ is of order 0.1 to 0.01 times $\Delta$. (If the conversion factor between $\theta$ and $\Delta$ were much smaller, e.g. with yet lower atom density and, hence, lower $T_{c}$, the phenomena discussed below might be limited to inaccessibly small angles.)

Define a scaled temperature $\tau \equiv T / T_{c}$. Then $R(\Delta, \tau)$, the total scattering rate normalized to the Rayleigh rate off a density of $N_{\text {total }}$, is given by

$$
\begin{aligned}
R(\Delta, \tau)= & 1 \\
& +N_{\text {total }}^{-1} \int \frac{d^{3} \mathbf{p} d^{3} \mathbf{p}^{\prime} \delta^{3}\left(\mathbf{p}-\mathbf{p}^{\prime}-\mathbf{\Delta}\right)}{\left[\lambda^{-1}(\tau) e^{p^{2} / 2 \tau}-1\right]\left[\lambda^{-1}(\tau) e^{p^{2} / 2 \tau}-1\right]} \\
& +2 \cdot \frac{N_{o}(\tau)}{N_{\text {total }}} \int \frac{d^{3} \mathbf{p} d^{3} \mathbf{p}^{\prime} \delta^{3}\left(\mathbf{p}^{\prime}\right) \delta^{3}\left(\mathbf{p}-\mathbf{p}^{\prime}-\mathbf{\Delta}\right)}{\left[\lambda^{-1}(\tau) e^{p^{2} / 2 \tau}-1\right]}
\end{aligned}
$$

where

$$
\begin{gathered}
N_{\text {total }}=\int d^{3} \mathbf{p}\left(e^{p^{2} / 2}-1\right)^{-1}=\frac{1}{2}(2 \pi)^{3 / 2} \zeta\left(\frac{3}{2}\right) \\
N_{o}(\tau)=\left(1-\tau^{3 / 2}\right) N_{\text {total }} \Theta(1-\tau) \\
\lambda(\tau) \equiv e^{\mu(\tau) / \tau} \\
=1-\left\{\frac{9}{16 \pi}\left[\zeta\left(\frac{3}{2}\right)\right]^{2}(\tau-1)^{2}+\mathcal{O}\left((\tau-1)^{3}\right)\right\} \Theta(\tau-1)
\end{gathered}
$$

and where $\mu(\tau)$ is the chemical potential and the Riemann $\zeta(3 / 2) \simeq 2.61$. The coefficient in $\lambda(\tau)$ of $(\tau-1)$ above $\tau=1$ is determined by requiring $N_{\text {total }}$ (which in these units is 
the critical density) to be independent of $\tau$. The virtue of the dimensionless variables $\Delta, \tau$, and $N_{\text {total }}$ is that a single function $R(\Delta, \tau)$ describes all physical situations, and interesting phenomena occur when the variables are roughly $\mathcal{O}(1)$. The actual light frequency, atom density, and atom mass then determine the translation of $(\Delta, \tau)$ into $(\theta, T)$.

The integral in term (2b) cannot be evaluated in closed form. After using the $\delta$-function and integrating over angles, one is faced with a one-dimensional integral, depending on $\Delta$ and $\tau$, that must be performed numerically. Before addressing the results, note that, despite the $\Theta$-functions in $N_{o}$ and $\lambda$, both $R(\Delta, \tau)$ and $\partial R / \partial \tau$ are continuous functions of $\tau$. (The latter is not immediately obvious but can be confirmed by an explicit evaluation of the contribution to $\partial R / \partial \tau$ from the $\tau$-dependence of $\lambda(\tau)$ at $\tau=1$; this can be done analytically. The discontinuity in this contribution to the $\tau$ derivative is exactly cancelled by the $\tau$-dependence of term (2c). The $\tau$-dependence arising from the $e^{p^{2} / \tau}$ 's is clearly continuous.)

The Bose final state enhancement of the total scattering, i.e., integrated over all angles, is a factor of $N_{\text {total }}$, independent of $\tau$. What changes with $\tau$ is the angular dependence of that enhancement. As $\tau$ decreases, it is concentrated at smaller and smaller angles - because the thermal distribution is at lower and lower momenta. What happens as we go below $\tau=1$ is that the finite fraction of the Bose enhancement involving the $p=0$ Bose-Einstein condensate has a different angular distribution, concentrated at somewhat smaller angles. It is the singular nature of the Bose distribution as $\mathbf{p} \rightarrow 0$ and $\tau \rightarrow 1^{+}$that keeps $R$ and $\partial R / \partial \tau$ formally continuous. Nevertheless, the numerical evaluation reveals a dramatic crossover for sufficiently small $\Delta$.

The most interesting ranges of parameters are displayed in figure (1). Above the critical temperature, $\Delta$ 's of order 1 or below show a Bose enhancement (solid lines) of a factor of two to five. Below the critical temperature, for $\Delta$ 's like 0.1 or below, the enhancement can jump to several hundred or thousand with only a ten to twenty percent drop in temperature. The dashed curves are the contribution to the normalized rate coming from scatterings involving the $p=0$ states, either as targets or final states. These scatterings account for a substantial 
fraction but by no means all of the enhancement. At fixed $\Delta$, as $T \rightarrow 0$, the enhancement must ultimately disappear. (It is then concentrated into yet smaller angles).

The above effects are really just simple kinematics that follow from the Bose occupation numbers. However, they have a potential practical application in cold atom optics. By collecting scattered light at small angles, one could significantly reduce the incident intensity needed to produce a given signal strength. The thermal average of the enhanced small angle scatterings do not heat the gas at all. They collectively remove precisely as much energy as they add because they involve all possible transitions from the thermal distribution back into the thermal distribution. The isotropic component (2a) is always present, and it does always contribute to heating. However, if the rate at some milliradians is 500 times greater than at 90 degrees, then one could reduce the incident intensity and, hence, total heating rate by 500 by moving the detector to small angles and still have the same signal strength.

The present discussion makes the simplifying assumption that the target gas is uniform over the beam size. One practical effect of finite sample size is that diffraction limits the smallest meaningful angles. For current experiments [2], the conversion of $\Delta$ to a laboratory scattering angle puts the interesting angles at the border of what might be observed. A small atom trap may also produce significant variation in the position space support of the wave functions. This can be used, of course, to advantage in identifying the ground state occupancy [2]. The formulae presented here are more appropriate to a trap that is large compared to the beam size or more like an ideal box. Nevertheless, the behavior for small, harmonic traps should be similar [1]. There are again three classes of states: unoccupied high energy states, a thermal occupation distribution of low energy excited states, and, finally, the ground state, with a significant occupancy. And the "unstimulated" rate is linear in the density while the stimulated rate is quadratic. One salient difference between trapped atoms and a uniform medium is that, with an external potential, momentum is not conserved [1]. In an infinite, uniform medium, the super-stimulated ground-state-to-groundstate transitions correspond to zero photon scattering angle. The corresponding transitions in a trap are analogs of the Mössbauer effect and are dominant for photon momentum 
transfers up to about double the largest typical ground state atom momentum. It is only beyond the corresponding maximum scattering angle that trap ground-state-to-excited-state and excited-state-to-excited-state scattering are the largest effects. Note, however, that if the light scattering is performed after a significantly long time interval of free expansion, i.e. after suddenly turning off the trapping fields [2], the Bose-stimulated enhancement will be confined to increasinly smaller angles. This is because, under free propagation, different momentum components become spatially separated. So the overlap between the scattered atom, of a particular final momentum, and previously existing atoms with that same momentum decreases rapidly with time.

Many recent theoretical discussions of optical effects in low temperature Bose gases have focused on far subtler issues, such as coherence in forward scattering at low [3] or high [4,5] densities (relative to the optical wavelength) or with pulsed light [6], or the effects of finite trap size [1] or transition line shapes [0]. (For a comprehensive review, see [8].) Although the fact that stimulated scattering occurs at small angles has often been mentioned in passing, a quantitative estimate of the magnitudes, angles, and sharpness with temperature of the effect had not been given. As emphasized in this context by Javanainen [9], to manifest any dramatic consequence of Bose statistics, the situation must involve a substantial number of identical particles that can be in the same place. For the present discussion to be relevant, the figure of merit is the number of atoms in the volume defined by the inverse of the momentum transfer.

\section{ACKNOWLEDGMENTS}

The author thanks K. Libbrecht and J. Preskill for several very helpful observations and suggestions. This work was supported in part by the U.S. Dept. of Energy under Grant No. DE-FG03-92-ER40701. 


\section{REFERENCES}

[1] J. Javanainen, Phys. Rev. Lett. 72, 2375 (1993).

[2] M.H. Anderson, J.R. Ensher, M.R. Matthews, C.E. Wieman, and E.A. Cornell, Science 269, 198 (1995).

[3] O. Morice, Y. Castin, and J. Dalibard, Phys. Rev. A 51, 3896 (1995).

[4] B.V. Svistunov and G.V. Shlyapnikov, Sov. Phys. JETP 70, 460 (1990); 71, 71 (1990).

[5] H. D. Politzer, Phys. Rev. A 43, 6444 (1991).

[6] M. Lewenstein and L. You, Phys. Rev. Lett. 71, 1339 (1993).

[7] L. You, M. Lewenstein, and J. Cooper, Phys. Rev. A 50, R3565 (1994).

[8] M. Lewenstein, L. You, J. Cooper, and K. Burnett, Phys. Rev. A 50, 2207 (1994).

[9] J. Javanainen, unpublished. 


\section{FIGURES}

FIG. 1. The scattered light, total enhancement factor, $R\left(\Delta, T / T_{c}\right)$, including Bose stimulated atomic motion (solid lines) versus temperature at different reduced angles $\Delta$ (see eqn. (1)). The dashed curves are the condensate contributions (i.e., from eq. (2c)). 


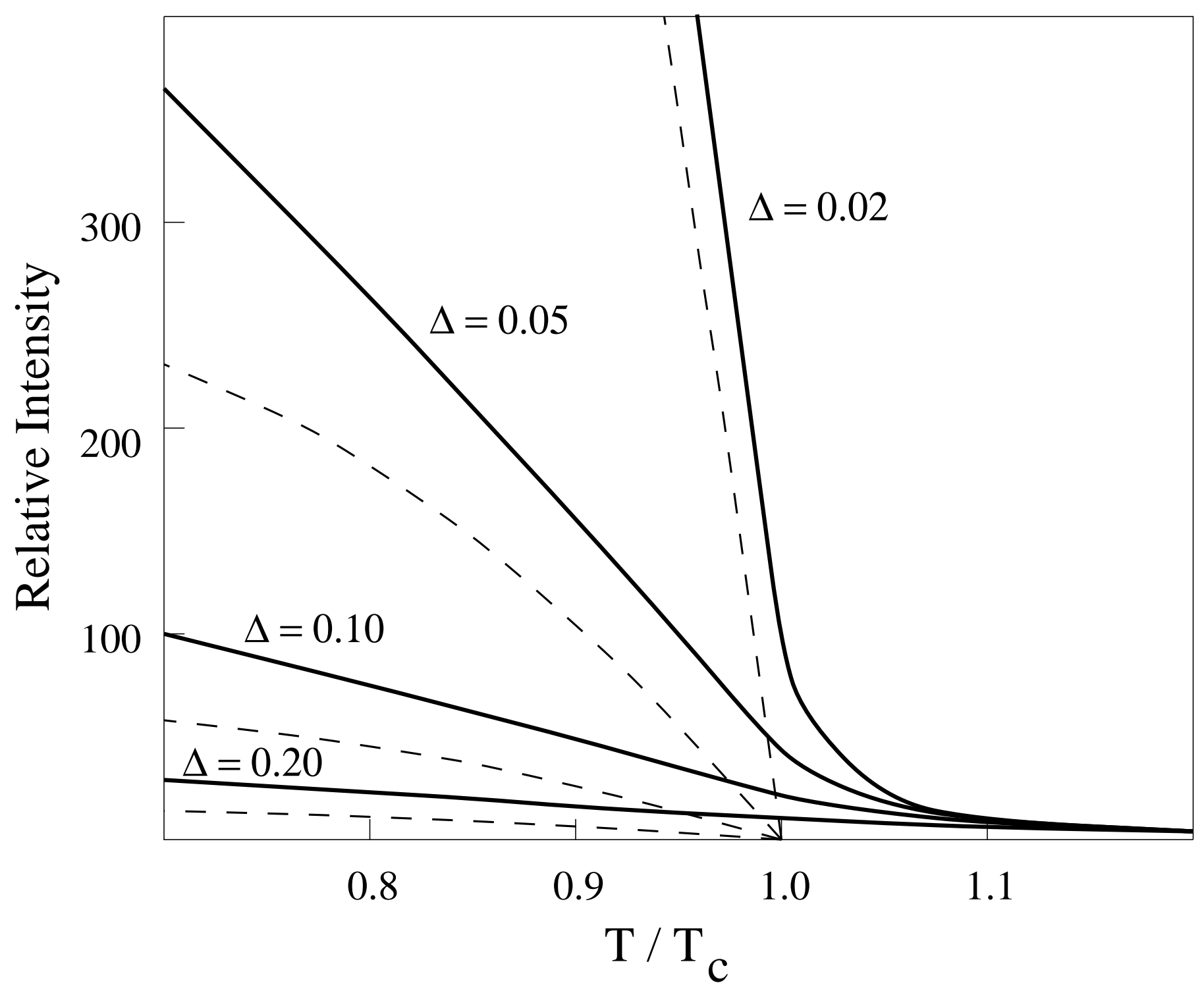

